\title{
UNIFORM STABILIZATION OF THE EULER-BERNOULLI EQUATION WITH FEEDBACK OPERATOR ONLY IN THE NEUMANN BOUNDARY CONDITION
}

\author{
N. Ourada And R. Triggiani \\ Department of Applied Mathematics, University of Virginia \\ Charlottesville, Virginia 22903
}

(Submitted by: A. V. Balakrishnan)

\begin{abstract}
We study the uniform stabilization problem for the Euler-Bernoulli equation defined in a smooth, bounded domain $\Omega$ of $\mathbb{R}^{n}$, with just one suitable dissipative boundary feedback operator acting on the Neumann B.C., while the Dirichlet B.C. is kept homogeneous. The uniform stabilization results which we present are fully consistent with recently established exact controllability and optimal regularity theories, which in fact motivate the choice of the function spaces in the first place. In particular, if the dissipative feedback operator acts on the entire boundary $\Gamma$, no geometrical conditions on $\Omega$ are needed.
\end{abstract}

\section{Introduction, preliminaries, and statement of main results.}

1.1 Introduction and Literature. Let $\Omega$ be an open, bounded domain in $\mathbb{R}^{n}$, where typically $n \geq 2$, with sufficiently smooth boundary $\Gamma=\Gamma_{0} \cup \Gamma_{1}, \Gamma_{i}$ relatively open with $\Gamma_{0}$ possibly empty, while $\Gamma_{1}$ is non-empty.

In $\Omega$ we consider the Euler-Bernoulli mixed problem in $w(t, x)$ on an arbitrary time interval $(0, T]$ with homogeneous Dirichlet boundary condition and non-homogeneous forcing term (control function) acting only in (possibly a part of) the Neumann boundary condition:

$$
\begin{cases}w_{t t}+\Delta^{2} w=0 & \text { in }(0, T] \times \Omega=Q ; \\ w(0, \cdot)=w_{0}, w_{t}(0, \cdot)=w_{1} & \text { in } \Omega ; \\ \left.w\right|_{\Sigma} \equiv 0 & \text { in }(0, T] \times \Gamma=\Sigma ; \\ \left.\frac{\partial w}{\partial \nu}\right|_{\Sigma_{0}} \equiv 0 & \text { in }(0, T] \times \Gamma_{0}=\Sigma_{0} \\ \left.\frac{\partial w}{\partial \nu}\right|_{\Sigma_{1}}=g & \text { in }(0, T] \times \Gamma_{1}=\Sigma_{1} .\end{cases}
$$

Data $\left\{w_{0}, w_{1}, g\right\}$ in $L_{2}(\Omega) \times H^{-2}(\Omega) \times L_{2}(\Sigma)$ produce a unique solution $\left\{w, w_{t}\right\} \in$ $C\left([0, T] ; L_{2}(\Omega) \times H^{-2}(\Omega)\right)[6]$, [7], an optimal regularity result. Indeed, exact controllability on $[0, T], T>0$ arbitrary, on the state space $L_{2}(\Omega) \times H^{-2}(\Omega)$ within the class of $L_{2}(\Sigma)$-controls $g$ holds true as well [6], [7]. It should be noted at the outset that the case where a control function acts in the Dirichlet B.C. (1.1c), while the control $g$ in (1.1e) may or may not be zero has also been studied and is, in fact, quite different from the case (1.1) of the present paper (the function spaces are different, the multipliers are different): here results of exact controllability on

Received in revised January 15, 1990

AMS Subject Classifications: 35B37. 
an arbitrary $T>0$ on (appropriate) spaces of optimal regularity are obtained in $[8],[9],[10]$, while results of uniform stabilization on such spaces are obtained in [1], by means of an explicit, dissipative feedback acting on the velocity $w_{t}$. Finally, we remark that the abstract theory of the linear quadratic regulator problem and corresponding algebraic Riccati equation as in [2] (which extends the case of the wave equation with Dirichlet control as in [11]) covers both the above problem (1.1), as well as the case of a control function acting in the Dirichlet B.C. (1.1c). As a result, it produces in both cases a feedback operator, based on the algebraic Riccati operator acting on the full pair $\left\{w, w_{t}\right\}$, which yields uniform stabilization in the spaces of optimal regularity and exact controllability (see [2; Appendix D] and [12]). Indeed, it is precisely the foregoing theory of exact controllability on spaces of optimal regularity which guarantees the Finite Cost Condition of the quadratic cost problem over an infinite horizon, and thus allows for the application of the Riccati theory in [2].

One problem that still needs investigation to complete the overall theory is the problem of uniform stabilization of the dynamics (1.1) by means of an explicit, dissipative feedback operator based on $w_{t}$. The present paper is devoted precisely to this problem. The results which we shall obtain in Theorem 1.2 are fully consistent with recent results of exact controllability [6], [7], obtained directly rather than through stabilization, both in regard to the spaces of optimal regularity and in regard to the properties of the triple $\left\{\Omega, \Gamma_{0}, \Gamma_{1}\right\}$; in particular, in regard to the lack of geometrical conditions on $\Omega$ (except for smoothness of $\Gamma$ ), if $g$ in (1.1e) is applied to all of $\Gamma$ (i.e., $\Gamma_{0}=\emptyset$ ). Indeed, according to a well-known result for time reversible systems [16], the uniform stabilization results given here imply corresponding exact controllability results: these precisely coincide with those in [6], [7] as far as spaces and properties of the triple $\left\{\Omega, \Gamma_{0}, \Gamma_{1}\right\}$ in terms of a radial field are concerned. However, in Remarks 1.2 and 1.3 we point out possible generalizations of our uniform stabilization result (hence of exact controllability) which involve suitably small perturbations of a radial field.

1.2. Preliminaries and choice of dissipative feedback. Throughout this paper, we let $A: L_{2}(\Omega) \supset \mathcal{D}(A) \rightarrow L_{2}(\Omega)$ be the positive self-adjoint operator defined by

$$
A f=\Delta^{2} f, \mathcal{D}(A)=H^{4}(\Omega) \cap H_{0}^{2}(\Omega) .
$$

We have [3]

$$
\mathcal{D}\left(A^{1 / 4}\right)=H_{0}^{1}(\Omega) ; \mathcal{D}\left(A^{1 / 2}\right)=H_{0}^{2}(\Omega)=\left\{f \in H^{2}(\Omega):\left.f\right|_{\Gamma}=\left.\frac{\partial f}{\partial \nu}\right|_{\Gamma}=0\right\}
$$

with equivalent norms. Thus, for $f \in \mathcal{D}\left(A^{1 / 4}\right)=H_{0}^{1}(\Omega)$,

$$
\begin{aligned}
& \|f\|_{\mathcal{D}\left(A^{1 / 4}\right)}=\left\|A^{1 / 4} f\right\|_{L^{2}(\Omega)}, \quad \text { equivalent to }\|f\|_{H^{1}(\Omega)} \\
& \text { in turn equivalent to }\left\{\int_{\Omega}|\nabla f|^{2} d \Omega\right\}^{1 / 2}
\end{aligned}
$$

by Poincaré's inequality. Also, for $f \in \mathcal{D}\left(A^{1 / 2}\right)=H_{0}^{2}(\Omega)$,

$$
\|f\|_{\mathcal{D}\left(A^{1 / 2}\right)}=\left\|A^{1 / 2} f\right\|_{L_{2}(\Omega)}=\left\{\int_{\Omega}|\Delta f|^{2} d \Omega\right\}^{1 / 2} .
$$


As mentioned in the introduction, our optimal space will be

$$
Z=L_{2}(\Omega) \times H^{-2}(\Omega)=L_{2}(\Omega) \times\left[\mathcal{D}\left(A^{1 / 2}\right)\right]^{\prime} .
$$

1.3. Choice of feedback operator. With $g=0$ in (1.1e), the resulting homogeneous problem generates a unitary group on $L_{2}(\Omega) \times\left[\mathcal{D}\left(A^{1 / 2}\right)\right]^{\prime}$ described by the map $\left\{w_{0}, w_{1}\right\} \rightarrow\left\{w, w_{t}\right\}$. It is justified in Section 1.5 , see below (1.32), that the following choice of a feedback operator $\mathcal{F}\left(w_{t}\right)$ on $\Sigma_{1}=(0, \infty) \times \Gamma_{1}$,

$$
\left.\frac{\partial w}{\partial \nu}\right|_{\Sigma_{1}}=g=\mathcal{F}\left(w_{t}\right)=-\tilde{G}_{2}^{*} w_{t}=-\tilde{G}_{2}^{*} A A^{-1} w_{t}=\left[\Delta A^{-1} w_{t}\right]_{\Sigma_{1}}
$$

provides a reasonable candidate for the uniform stabilization problem of (1.1), in the sense that the closed loop feedback dynamics with (1.7) used as (1.1e) is wellposed in the semigroup sense in $Z$, and all of its solutions originating in $Z$ decrease as $t \rightarrow \infty$ in the $Z$-norm. To show that they decrease to zero, and in fact, in the uniform norm $\mathcal{L}(Z)$, is the major task of this paper. In (1.7) we have set $\tilde{G}_{2}$ to be the operator (Green map):

$$
\begin{gathered}
\tilde{G}_{2} g_{2}=y \Longleftrightarrow \begin{cases}\Delta^{2} y=0 & \text { in } \Omega ; \\
\left.y\right|_{\Gamma}=0 & \text { in } \Gamma ; \\
\left.\frac{\partial y}{\partial \nu}\right|_{\Gamma_{0}}=0,\left.\frac{\partial y}{\partial \nu}\right|_{\Gamma_{1}}=g_{2} ;\end{cases} \\
\tilde{G}_{2}: L_{2}(\Gamma) \rightarrow H^{3 / 2}(\Omega) \cap H_{0}^{1}(\Omega),
\end{gathered}
$$

while $\tilde{G}_{2}^{*}$ is the adjoint of $\tilde{G}_{2}$ in the sense that

$$
\left(\tilde{G}_{2} g_{2}, v\right)_{L_{2}(\Omega)}=\left(g_{2}, \tilde{G}_{2}^{*} v\right)_{L_{2}\left(\Gamma_{1}\right)}, \forall g_{2} \in L_{2}\left(\Gamma_{1}\right), v \in L_{2}(\Omega) .
$$

Moreover, it is proved by Green's theorem that [9]

$$
\tilde{G}_{2}^{*} A f=\left\{\begin{array}{ll}
0 & \text { in } \Gamma_{0} \\
-[\Delta f]_{\Gamma_{1}} & \text { in } \Gamma_{1}
\end{array} f \in \mathcal{D}(A) .\right.
$$

Thus, (1.11) justifies the last step in (1.7).

1.4. The feedback system: Statement of main results. By virtue of (1.7), the resulting candidate feedback system, whose stability properties in $Z$ we shall investigate, is

$$
\begin{cases}w_{t t}+\Delta^{2} w=0 & \text { in }(0, T) \times \Omega=Q \\ w(0, \cdot)=w_{0}, \quad w_{t}(0, \cdot)=w_{1} & \text { in } \Omega \\ \left.w\right|_{\Sigma} \equiv 0 & \text { in }(0, T) \times \Gamma=\Sigma \\ \left.\frac{\partial w}{\partial \nu}\right|_{\Sigma_{0}} \equiv 0 & \text { in }(0, T) \times \Gamma_{0}=\Sigma_{0} \\ \left.\frac{\partial w}{\partial \nu}\right|_{\Sigma_{1}}=\left[\Delta\left(A^{-1} w_{t}\right)\right]_{\Sigma_{1}} & \text { in }(0, T) \times \Gamma_{1}=\Sigma_{1}\end{cases}
$$

Using the techniques of [18], [14], [9], [1], etc., problem (1.12) can be rewritten more conveniently in abstract form as

$$
w_{t t}=-A w-A \tilde{G}_{2} \tilde{G}_{2}^{*} w_{t}
$$




$$
\begin{gathered}
\frac{d}{d t}\left|\begin{array}{c}
w \\
w_{t}
\end{array}\right|=\mathcal{A}\left|\begin{array}{c}
w \\
w_{t}
\end{array}\right| \text { on } Z ; \\
\mathcal{A}=\left|\begin{array}{cc}
0 & I \\
-A & -A \tilde{G}_{2} \tilde{G}_{2}^{*}
\end{array}\right|: \mathcal{D}(\mathcal{A})=\{y \in Z: \mathcal{A} y \in Z\} .
\end{gathered}
$$

A more explicit description of $\mathcal{D}(\mathcal{A})$ will be given in the subsequent analysis; see (2.3)-(2.5), below.

Theorem 1.1. (i) (Well-posedness on $Z$ ) The operator $\mathcal{A}$ in (1.14) is dissipative on $\mathcal{D}(\mathcal{A}) \subset Z=L_{2}(\Omega) \times\left[\mathcal{D}\left(\mathcal{A}^{1 / 2}\right)\right]^{\prime}$ (see (1.6)) and satisfies here the range condition: range $(\lambda I-\mathcal{A})=Z$ for all $\lambda>0$. Thus, by Lumer-Phillips Theorem, $\mathcal{A}$ generates a strongly continuous contraction semigroup $e^{\mathcal{A} t}$ on $Z$. The resolvent operator $R(\lambda, \mathcal{A})$ is given by

$$
\begin{gathered}
R(\lambda, \mathcal{A})=\left[\begin{array}{cc}
\frac{I-V^{-1}(\lambda)}{\lambda} & V^{-1}(\lambda) A^{-1} \\
-V^{-1}(\lambda) & \lambda V^{-1}(\lambda) A^{-1}
\end{array}\right] \\
V(\lambda)=I+\lambda \tilde{G}_{2} \tilde{G}_{2}^{*}+\lambda^{2} A^{-1},
\end{gathered}
$$

at least for $\operatorname{Re} \lambda>0$; moreover, $R(\lambda, \mathcal{A})$ is compact on $Z$. The resolvent set of $\mathcal{A}$ satisfies $\rho(\mathcal{A}) \supset\{\lambda: \operatorname{Re} \lambda \geq 0\}$ if $\Gamma_{1}$ satisfies the uniqueness property (1.21) in Remark 1.1 below, which is certainly the case if $\Gamma_{1}=\Gamma$.

(ii) ( $L_{2}$-boundedness in time of the feedback operator) For $\left\{w_{0}, w_{1}\right\} \in Z$ the solution $w$ of (1.12) or (1.13) satisfies (see (1.7))

$$
\begin{gathered}
\frac{d E(t)}{d t}=-2 \int_{\Gamma_{1}}\left(\frac{\partial w}{\partial \nu}\right)^{2} d \Gamma=-2\left\|\tilde{G}_{2}^{*} w_{t}\right\|_{L_{2}\left(\Gamma_{1}\right)}^{2}=-2\left\|\Delta\left(A^{-1} w_{t}\right)\right\|_{L_{2}\left(\Gamma_{1}\right)}^{2} \leq 0 \\
E(t)-E(0)=-2 \int_{0}^{t} \int_{\Gamma_{1}}\left(\frac{\partial w}{\partial \nu}\right)^{2} d \Gamma d t=-2 \int_{0}^{t}\left\|\tilde{G}_{2}^{*} w_{t}\right\|_{L_{2}\left(\Gamma_{1}\right)}^{2} d t ; \\
\int_{0}^{\infty} \int_{\Gamma_{1}}\left(\frac{\partial w}{\partial \nu}\right)^{2} d \Gamma d t=\int_{0}^{\infty}\left\|\tilde{G}_{2}^{*} w_{t}\right\|_{L_{2}\left(\Gamma_{1}\right)}^{2} d t \leq E(0)
\end{gathered}
$$

where throughout the paper we set for convenience

$$
E(t) \equiv\left\|e^{\mathcal{A} t}\left|\begin{array}{l}
w_{0} \\
w_{1}
\end{array}\right|\right\|_{L_{2}(\Omega) \times\left[\mathcal{D}\left(A^{1 / 2}\right)\right]^{\prime}}^{2}=\|w(t)\|_{L_{2}(\Omega)}^{2}+\left\|A^{-1 / 2} w_{t}(t)\right\|_{L_{2}(\Omega)}^{2} .
$$

Remark 1.1. The point $i \mu, \mu$ real and $\mu \neq 0$, of the imaginary axis belongs to the resolvent set $\rho(\mathcal{A})$ of the operator $\mathcal{A}$ in (1.14), provided the following uniqueness property holds true: If $\phi(x)$ is a (smooth) function which satisfies

$$
\begin{aligned}
\Delta^{2} \phi & =\mu^{2} \phi & & \text { in } \Omega ; \\
\left.\phi\right|_{\Gamma} & =\left.\frac{\partial \phi}{\partial \nu}\right|_{\Gamma}=0 & & \text { in } \Gamma ; \\
\left.\Delta \phi\right|_{\Gamma_{1}} & =0 & & \text { in } \Gamma_{1} ;
\end{aligned}
$$

then, in fact, (see subsequent Section 1.5) 


$$
\phi \equiv 0 \quad \text { in } \Omega .
$$

Plainly, the above uniqueness holds true if $\Gamma_{1}=\Gamma$ : in this case, one readily obtains also the fourth boundary condition $\left.\frac{\partial(\Delta \phi)}{\partial \nu}\right|_{\Gamma}=0$ from the second condition in (1.21b), see, e.g., [20, Appendix A], and a standard uniqueness result yields the conclusion (1.21d).

More generally, a sufficient condition on the inactive portion of the boundary $\Gamma_{0}$ for the uniqueness (1.21) to hold true is that $\Gamma_{0}$ be as in (1.23) below (see subsequent Section 1.5).

The next theorem gives a uniform stabilization result, in particular when the feedback $(1.12 \mathrm{e})$ is active on the entire boundary $\Gamma$; i.e., when $\Gamma_{0}=\emptyset$. If instead $\Gamma_{0} \neq \emptyset$, then $\Gamma_{0}$ is assumed to be of the special form as in (1.23) below. Theorem 1.2 then recovers the exact controllability results obtained directly in [7] (same spaces and same assumption on $\Gamma_{0}$ ), by a direct application of [16].

Theorem 1.2. (Uniform stabilization: the radial field case).

(a) Let $\Gamma_{0}=\emptyset$ so that the feedback (1.12e) acts on all of $\Gamma$. Then, the feedback system (1.12), equivalently the abstract system (1.13), is uniformly (exponentially) stable on the space $Z$ given by (1.6): there exist constants $\delta>0$ and $M=M_{\delta} \geq 1$ such that

$$
\left\|\begin{array}{c}
w(t) \\
w_{t}(t)
\end{array}\left|\left\|_{Z}=\right\| e^{\mathcal{A} t}\right| \begin{array}{c}
w_{0} \\
w_{t}
\end{array}\left|\left\|_{Z} \leq M e^{-\delta t}\right\|\right| \begin{array}{c}
w_{0} \\
w_{1}
\end{array} \mid\right\|_{Z}, t \geq 0 .
$$

(b) More generally, the uniform decay (1.22) holds true for (1.12) if we take

$$
\Gamma_{0}=\Gamma_{-}\left(x_{0}\right)=\left\{x \in \Gamma:\left(x-x_{0}\right) \cdot \nu \leq 0\right\}
$$

for some point $x_{0} \in \mathbb{R}^{n}$, where $\nu=$ unit normal vector pointed outward.

Remark 1.2. The uniform stabilization result (1.22) in Theorem 1.2 may be (slightly) generalized to linear vector fields

$$
h_{i}(x)=a_{i}\left(x_{i}-x_{0, i}\right) \text { for some } x_{0}=\left[x_{0,1}, \ldots, x_{0, n}\right] \in \mathbb{R}^{n},
$$

where the coefficients $\left\{a_{i}\right\}$ are constant, and there is a constant $m>0$ such that the corresponding differences satisfy the condition that

$$
\sup _{i}\left|a_{i}-m\right|
$$

is sufficiently small. See Section 2.5. A further generalization is pointed out in the subsequent remark.

Remark 1.3. The uniform stabilization result (1.22) in Theorem 1.2 may be generalized to the case of a triplet $\left\{\Omega, \Gamma_{0}, \Gamma_{1}\right\}$ satisfying: there exists a vector field $h(x)=\left[h_{1}(x), \cdots, h_{n}(x)\right] \in\left[C^{3}(\bar{\Omega})\right]^{n}$ such that

(i)

$$
\int_{\Omega} \Delta q\left(\sum_{i=1}^{n} \nabla h_{i} \cdot \nabla q_{x_{i}}\right) d \Omega \geq \rho \int_{\Omega}(\Delta q)^{2} d \Omega
$$


where $q(x)$ is an $H_{0}^{2}(\Omega)$-function on $\Omega$ satisfying therefore

$$
\left.q\right|_{\Gamma}=\left.\frac{\partial q}{\partial \nu}\right|_{\Gamma}=0
$$

and $\rho>0$ is a suitable constant, possibly depending on $h(x), \Omega$, and $q(x)$;

(ii) either the (elliptic) uniqueness property (1.21) holds true, or else the corresponding dynamical uniqueness property: if $\psi(t, x)$ solves

then, in fact,

$$
\begin{array}{ll}
\psi_{t t}+\Delta^{2} \psi=0 & \text { in }(0, T] \times \Omega=Q \\
\left.\psi\right|_{\Sigma}=\left.\frac{\partial \psi}{\partial \nu}\right|_{\Sigma}=0 & \text { in }(0, T] \times \Gamma=\Sigma \\
\left.\Delta \psi\right|_{\Sigma_{1}}=0 & \text { in }(0, T] \times \Gamma_{1}=\Sigma_{1}
\end{array}
$$

$$
\psi \equiv 0 \quad \text { in } Q
$$

Either uniqueness property (1.21) or (1.28) holds true in case $\Gamma_{0}$ is given by (1.23).

The linear vector field in (1.24), (1.25) satisfies the inequality (1.26), and, moreover, the quantity ( $\max$ as $x$ varies in $\bar{\Omega}$ )

$$
M_{h}=\max \left\{\left|\Delta h_{1}\right|,|\Delta(\operatorname{div} h)|,|\nabla(\operatorname{div} h)|\right\}
$$

is zero in this case. Thus (see section 2.5) no lower order terms are involved in inequality (2.8) below: it is precisely to absorb the lower order term $\||\nabla p|\|_{C\left([0, T] ; L_{2}(\Omega)\right)}$ of inequality (2.8) that condition (ii) is invoked. More general perturbations of the radial field than the linear field $(1.24),(1.25)$ can be given which satisfy inequality (1.26), but then the uniqueness property (1.21) or (1.28) comes into play; see Section 2.5.

Remark 1.4. (i) Under the conditions of Theorem 1.2 , and also for the more general case described in Remark 1.2, explicit values may be computed for the constant $\delta$ in (1.22); in fact, $\delta=\frac{1}{t} \log \frac{1}{r_{T}}$, where $r_{T}=C_{T}\left(C_{T}+2\right)^{-1}$ and $C_{T}$ is as in (2.2). The constant $C_{T}$ can be estimated explicitly in these cases because the linearity of $h$ simplifies the integral identities used to prove (2.2).

(ii) In contrast, the extension described in Remark 1.3 assumes a general (nonlinear) $h$; the identities then contain terms of lower order in the dependent variable, which must be absorbed through a compactness/uniqueness argument by contradiction (Lemma 2.1 of Section 2.5). Thus, in this case, the constant $C_{T}$, hence the constant $\delta$, are not explicitly estimated.

The availability of an explicit $\delta$ therefore rests on whether the vector field $h$ assumed to describe $\Omega$ is linear, regardless of whether we use the subsequent criterion (2.1), or else Datko's theorem of (2.56) below, as done in [1].

1.5. Sketch of proof of Theorem 1.1: The proof of much of Theorem 1.1 is very similar to the proof of analogous results for wave equations with Dirichlet control [13], or Neumann control [19], and for Euler-Bernoulli equations with control in the Dirichlet B.C. [1]. Thus, details are omitted and only some distinctive features of problem (1.12) will be mentioned. According to techniques as in [18], [14], etc., for 
waves, or [1], [9], etc., for Euler-Bernoulli problem, the abstract differential equation which models problem (1.1) is

$$
w_{t t}=-A w+A \tilde{G}_{2} g
$$

(recall (1.8)) whose corresponding first-order system is

$$
\frac{d}{d t}\left|\begin{array}{c}
w \\
w_{t}
\end{array}\right|=\left|\begin{array}{cc}
0 & I \\
-A & 0
\end{array}\right|\left|\begin{array}{c}
w \\
w_{t}
\end{array}\right|+\left|\begin{array}{c}
0 \\
A \tilde{G}_{2} g
\end{array}\right|
$$

Since $\left|\begin{array}{cc}0 & I \\ -A & 0\end{array}\right|$ is skew-adjoint on $Z,(1.6)$, Eq. (1.31) plainly suggests to take $g=$ $-\tilde{G}_{2}^{*} w_{t}$ (modulo a positive function which we shall take identically 1 ) for feedback stabilization (as anticipated in (1.7)), for this choice then makes the corresponding feedback operator $\mathcal{A}$ in (1.14) dissipative on $Z$; indeed, for $y=\left[y_{1}, y_{2}\right] \in \mathcal{D}(\mathcal{A})$ :

$$
\begin{aligned}
\operatorname{Re}(\mathcal{A} y, y)_{Z} & =-\left(A \tilde{G}_{2} \tilde{G}_{2}^{*} y_{2}, y_{2}\right)_{\left[\mathcal{D}\left(A^{1 / 2}\right)\right]^{\prime}}=-\left(\tilde{G}_{2} \tilde{G}_{2}^{*} y_{2}, y_{2}\right)_{L_{2}(\Omega)} \\
& =-\left\|\tilde{G}_{2}^{*} y_{2}\right\|_{L_{2}\left(\Gamma_{1}\right)}^{2} \leq 0
\end{aligned}
$$

The proof of Theorem 1.1 follows closely the above references see [20, Appendix $\mathrm{A}]$; in particular, for the compactness of $\mathbb{R}(\lambda, \mathcal{A})$ one may follow the argument of [1; Theorem 1.1, Step 2]. The only point which needs further explanation is the claim that the imaginary axis is in the resolvent set $\rho(\mathcal{A})$ of $\mathcal{A}$. That $0 \in \rho(\mathcal{A})$ is immediate, as the resolvent is compact.

Next, if $\mu \neq 0$ real, an argument as in the above references yields that if $i \mu$ is an eigenvalue of $\mathcal{A}$, then Eqs. (1.21a-b-c) hold true, and if the uniqueness property (1.21d) applies, then we have a contradiction [20]. To show the final statement of Remark 1.1-that the uniqueness property (1.21) holds true for $\Gamma_{0}$ as in (1.23)-we proceed as follows. We apply the multipliers $\left(x-x_{0}\right) \cdot \nabla \phi$ and $\phi$ to problem (1.21a). This produces (see subsequent section 2.2)

$$
\int_{\Gamma}(\Delta \phi)^{2}\left(x-x_{0}\right) \cdot \nu d \Gamma=4 \int_{\Omega}(\Delta \phi)^{2} d \Omega .
$$

Then, using (1.21c) and (1.23), we obtain

$$
0=\int_{\Gamma_{1}}(\Delta \phi)^{2}\left(x-x_{0}\right) \cdot \nu d \Gamma \geq \int_{\Gamma}(\Delta \phi)^{2}\left(x-x_{0}\right) \cdot \nu d \Gamma,
$$

and then $\Delta \phi \equiv 0$ in $\Omega$ follows from (1.33): this, along with the first condition in (1.21b) yields $\phi \equiv 0$ in $\Omega$ as desired.

\section{Proof of Theorem 1.2.}

2.1. Preliminaries and a change of variable $w \rightarrow p$. With reference to the "energy" $E(t)$ of the $w$-problem (1.12) defined in (1.20) our task is, as usual, to show that there exists a time $0<T<\infty$ such that

$$
E(T) \leq r E(0), r<1 ; \quad \text { or }\left\|e^{\mathcal{A} T}\right\|_{\mathcal{L}(Z)}<1,
$$


$Z=L_{2}(\Omega) \times\left[\mathcal{D}\left(A^{1 / 2}\right)\right]^{\prime}$, norm-equivalent to $L_{2}(\Omega) \times H^{-2}(\Omega)$, after which the uniform decay (1.22) is then established. To prove (2.1), it will suffice, as usual, to show that there exists a time $0<T<\infty$ and a corresponding constant $c_{T}>0$ such that

$$
E(T) \leq c_{T} \int_{0}^{T} \int_{\Gamma_{1}}\left(\frac{\partial w}{\partial \nu}\right)^{2} d \Gamma_{1} d t
$$

for (2.2), combined with the non-increasing property (1.17) of $E(t)$, will then yield (1.22). Our subsequent effort is aimed as establishing (2.2). To this end, we use the idea introduced in [13] which consists in lifting the low (though optimal) topology $L_{2}(\Omega) \times H^{-2}(\Omega)$ for the solution $\left\{w(t), w_{t}(t)\right\}$ of $(1.12)$ to the level $H_{0}^{2}(\Omega) \times L_{2}(\Omega)$ suitable for multiplier techniques for the pair $\left\{p(t), p_{t}(t)\right\}$, where $p$ is the dependent variable of a new problem. This idea was also successfully used in [1] in the study of uniform stabilization of problem (1.1) with feedback also in the Dirichlet B.C. (1.1c). (But the transformation $w \rightarrow p$ in [1] is different from the one in (2.8) below for the present problem (1.12), due to the different topologies involved; moreover, for the very same reasons the multipliers used in the $p$-problem in [1] are different from the multipliers used in the present paper.)

Unless otherwise noted, we take henceforth $\left\{w_{0}, w_{1}\right\} \in \mathcal{D}(\mathcal{A})$ and show the estimate (2.2) with constant $c_{T}$ independent of $\left\{w_{0}, w_{1}\right\}$. It follows readily from (1.14) that $\mathcal{A} z=\left\{z_{2},-A\left[z_{1}+\tilde{G}_{2} \tilde{G}_{2}^{*} z_{2}\right]\right\} \in Z=L_{2}(\Omega) \times\left[\mathcal{D}\left(A^{1 / 2}\right)\right]^{\prime}$ implies

$$
\left\{z_{1}, z_{2}\right\} \in \mathcal{D}(\mathcal{A}) \Longrightarrow\left\{\begin{array}{l}
z_{2} \in L_{2}(\Omega) ; \\
z_{1}+\tilde{G}_{2} \tilde{G}_{2}^{*} z_{2} \in \mathcal{D}\left(A^{1 / 2}\right)=H_{0}^{2}(\Omega),
\end{array}\right.
$$

and thus, by (1.9),

$$
\tilde{G}_{2} \tilde{G}_{2}^{*} z_{2} \in H^{3}(\Omega) \cap H_{0}^{1}(\Omega), \quad \text { hence } z_{1} \in H^{2}(\Omega) \cap H_{0}^{1}(\Omega),
$$

upon using (2.4). Since $\left\{w_{0}, w_{1}\right\} \in \mathcal{D}(\mathcal{A})$ implies $\left.\left\{w(t), w_{t}(t)\right\} \in C([0, T] ; \mathcal{D}(\mathcal{A})]\right)$ by Theorem 1.1(i), we then have by $(2.3)$ and $(2.5)$,

$$
\left|\begin{array}{l}
w_{0} \\
w_{1}
\end{array}\right| \in \mathcal{D}(\mathcal{A}) \Longrightarrow\left\{\begin{array}{l}
w(t) \in C\left([0, T] ; H^{2}(\Omega) \cap H_{0}^{1}(\Omega)\right) \\
w_{t}(t) \in C\left([0, T] ; L_{2}(\Omega)\right)
\end{array}\right.
$$

Following the idea in [13], [1], we then introduce a new variable $p$ in the present case by setting

$$
\begin{gathered}
A^{1 / 2} p=A^{-1 / 2} w_{t} ; \text { i.e., } \\
p=A^{-1} w_{t} \in \begin{cases}C\left([0, T] ; \mathcal{D}\left(A^{1 / 2}\right)\right) & \text { if }\left\{w_{0}, w_{1}\right\} \in Z ; \\
C([0, T] ; \mathcal{D}(A)) & \text { if }\left\{w_{0}, w_{1}\right\} \in \mathcal{D}(\mathcal{A}) .\end{cases}
\end{gathered}
$$

From (2.8) and (1.13a), we obtain

$p_{t}=A^{-1} w_{t t}=-w-\tilde{G}_{2} \tilde{G}_{2}^{*} w_{t} \in \begin{cases}L_{2}\left(0, T ; L_{2}(\Omega)\right) & \text { if }\left\{w_{0}, w_{1}\right\} \in Z \\ C\left([0, T] ; \mathcal{D}\left(A^{1 / 2}\right)\right) & \text { if }\left\{w_{0}, w_{1}\right\} \in \mathcal{D}(\mathcal{A}),\end{cases}$

where the regularity in (2.9) follows from (1.18), (2.6), and (2.4). Hence, from (2.9) we obtain via $(2.8)$ (left),

$$
p_{t t}=-w_{t}-\tilde{G}_{2} \tilde{G}_{2}^{*} w_{t t}=-A p-\tilde{G}_{2} \tilde{G}_{2}^{*} w_{t t} .
$$


In terms of the scalar function $p(t, x), x \in \Omega$, corresponding to the vector-valued $p(t)=p(t, \cdot)$, the abstract equation $(2.10)$ can be rewritten as the following EulerBernoulli problem:

$$
\left\{\begin{array}{l}
p_{t t}+\Delta^{2} p=F \\
p(0, \cdot)=p_{0}=A^{-1} w_{1} ; p_{t}(0, \cdot)=p_{1}=A^{-1} w_{t t}(0) \\
\left.p\right|_{\Sigma} \equiv 0 \\
\left.\frac{\partial p}{\partial \nu}\right|_{\Sigma} \equiv 0
\end{array}\right.
$$

where $p_{0} \in \mathcal{D}(A)$ and $p_{1}=-\left(w_{0}+\tilde{G}_{2} \tilde{G}_{2}^{*} w_{1}\right) \in \mathcal{D}\left(A^{1 / 2}\right)$, by $(2.8 \mathrm{~b})$ and $(2.9 \mathrm{~b})$, respectively, and where the homogeneous boundary conditions in $(2.11 \mathrm{c}-\mathrm{d})$ are a consequence of $p \in \mathcal{D}\left(A^{1 / 2}\right)=H_{0}^{2}(\Omega)$ from (2.8) and (1.3). In (2.11a) we have set

$$
F=-\tilde{G}_{2} \tilde{G}_{2}^{*} w_{t t}=-\tilde{G}_{2} \tilde{G}_{2}^{*} A A^{-1} w_{t t}=\tilde{G}_{2}\left[\Delta p_{t}\right]_{\Sigma_{1}}
$$

In the sequel, we shall have to consider pointwise values of $p_{t}(t)$ : these make sense by $(2.9 \mathrm{~b})$ for initial data $w_{0}, w_{1} \in \mathcal{D}(\mathcal{A})$ as assumed, while from $(2.9 \mathrm{a})$ the pointwise meaning of $p_{t}(t)$ in $L_{2}(\Omega)$ is lost for general initial data in $Z$. In the analysis below of the $p$-system (2.11), we shall crucially use from (2.8) (left) and (2.9) (left), respectively, and (1.5),

$$
\begin{gathered}
\left\|w_{t}\right\|_{\left[\mathcal{D}\left(A^{1 / 2}\right)\right]^{\prime}}=\left\|A^{-1 / 2} w_{t}\right\|_{L_{2}(\Omega)}=\left\|A^{1 / 2} p\right\|_{L_{2}(\Omega)}=\left\{\int_{\Omega}(\Delta p)^{2} d \Omega\right\}^{1 / 2} \\
p_{t}=-w+\mathcal{O}\left(\left\|\tilde{G}_{2}^{*} w_{t}\right\|_{L_{2}\left(\Gamma_{1}\right)}\right)
\end{gathered}
$$

where $\frac{\partial w}{\partial \nu}=-\tilde{G}_{2}^{*} w_{t} \in L_{2}\left(0, \infty ; L_{2}\left(\Gamma_{1}\right)\right)$ from (1.19), since $G_{2}$ is bounded on $L_{2}(\Gamma)$ (see (1.9)). Recalling the 'energy' $E(t)$ of the $w$-problem from (1.20), we have via (2.13), (2.14),

$$
E(t)=\int_{\Omega} p_{t}^{2}(t)+(\Delta p(t))^{2} d \Omega+\mathcal{O}\left(\left\|\tilde{G}_{2}^{*} w_{t}\right\|_{L_{2}\left(\Gamma_{1}\right)}^{2}\right) .
$$

In $(2.14),(2.15)$, the symbol $\mathcal{O}$ means, as usual, bounded above by a constant, in fact, independent of $T$. Dependence of constants on $T$ will always be noted explicitly.

2.2. Integral identities for the $p$-problem (2.11). Throughout, we let $Q=(0, T] \times \Omega, \Sigma=(0, T] \times \Gamma$, etc.

Proposition 2.1. Let $h(x)=\left[h_{1}(x), \cdots, h_{n}(x)\right] \in\left[C^{3}(\bar{\Omega})\right]^{n}$ be a given vector field, and let $\left\{w_{0}, w_{1}\right\} \in \mathcal{D}(\mathcal{A})$ so that $\left\{p_{0}, p_{1}\right\} \in \mathcal{D}(A) \times \mathcal{D}\left(A^{1 / 2}\right)$. Then the solution $p$ of problem (2.11) satisfies the following identity:

$$
\begin{aligned}
& \frac{1}{2} \int_{\Sigma}(\Delta p)^{2} h \cdot \nu d \Sigma=2 \int_{Q} \Delta p\left(\sum_{i=1}^{n} \nabla h_{i} \cdot \nabla p_{x_{i}}\right) d Q+\int_{Q} \Delta p\left[\Delta h_{1}, \cdots, \Delta h_{n}\right] \cdot \nabla p d Q \\
& \quad+\frac{1}{2} \int_{Q}\left[p_{t}^{2}-(\Delta p)^{2}\right] \operatorname{div} h d Q-\int_{Q} F h \cdot \nabla p d Q+\left[\left(p_{t}(t), h \cdot \nabla p(t)\right)_{\Omega}\right]_{0}^{T} .
\end{aligned}
$$


Remark 2.1. We note explicitly that the following identities hold true:

$$
\begin{aligned}
\operatorname{div}(H \nabla p) & =\sum_{i=1}^{n} \nabla h_{i} \cdot \nabla p_{x_{i}}+\nabla p \cdot \nabla(\operatorname{div} h) \\
\operatorname{div}\left(H^{T} \nabla p\right) & =\sum_{i=1}^{n} \nabla h_{i} \cdot \nabla p_{x_{i}}+\left[\Delta h_{1}, \cdots, \Delta h_{n}\right] \cdot \nabla p,
\end{aligned}
$$

where $H=H(x)$ is the $n \times n$ matrix with $(i, j)$-entry $\frac{\partial h_{i}}{\partial x_{j}}$ and $H^{T}$ its transpose, so that (2.17) and (2.18) imply

$\operatorname{div}\left[\left(H+H^{T}\right) \nabla p\right]=2 \sum_{i=1}^{n} \nabla h_{i} \cdot \nabla p_{x_{i}}+\nabla p \cdot \nabla(\operatorname{div} h)+\left[\Delta h_{1}, \cdots, \Delta h_{n}\right] \cdot \nabla p$

and hence $(2.16)$ can be rewritten as

$$
\begin{aligned}
& \frac{1}{2} \int_{\Sigma}(\Delta p)^{2} h \cdot \nu d \Sigma=\frac{1}{2} \int_{Q}\left[p_{t}^{2}-(\Delta p)^{2}\right] \operatorname{div} h d Q+\int_{Q} \Delta p \operatorname{div}\left[\left(H+H^{T}\right) \nabla p\right] d Q \\
& \quad-\int_{Q} \Delta p \nabla p \cdot \nabla(\operatorname{div} h) d Q-\int_{Q} F h \cdot \nabla p d Q+\left[\left(p_{t}(t), h \cdot \nabla p(t)\right)_{\Omega}\right]_{0}^{T} .
\end{aligned}
$$

Proof of Proposition 2.1: One uses the multiplier $h \cdot \nabla p$ as in [7], [4], [15], [19]. (We use a general field $h$, even though we shall specialize later to radial fields in our principal result, Theorem 1.2, mostly for the benefit of including in our arguments the generalizations pointed out in Remarks 1.2 and 1.3.) Details in [20].

We now handle the first integral on the right of $(2.20)$.

Proposition 2.2. Under the assumptions of Proposition 2.1, the solution $p$ of problem (2.11) satisfies the following identity:

$$
\begin{aligned}
& \int_{Q}\left[p_{t}^{2}-(\Delta p)^{2}\right] \operatorname{div} h d Q=-\int_{Q} F p \operatorname{div} h d Q+\int_{Q} p \Delta p \Delta(\operatorname{div} h) d Q \\
& \left.\quad+2 \int_{Q} \Delta p \nabla p \cdot \nabla(\operatorname{div} h) d Q+\left[\left(p_{t}(t), p(t) \operatorname{div} h\right)\right]_{\Omega}\right]_{0}^{T} .
\end{aligned}
$$

Remark 2.2. We note explicitly for future use that identity (2.21) continues to hold true if we set $\operatorname{div} h \equiv 1$; i.e., if in the proof we multiply Eq. (2.11a) simply by $p$ rather than $p \operatorname{div} h$.

Proof of Proposition 2.2: One uses the multiplier $p \operatorname{div} h$ [7], [4], [15]; see [20].

We next insert (2.21) into (2.16), or respectively, $(2.20)$, and obtain the final identity of the $p$-system.

Proposition 2.3. Under the assumptions of Propostion 2.1, the solution $p$ of (2.12) satisfies the following identity (from (2.16)):

$$
\begin{aligned}
\frac{1}{2} \int_{\Sigma}(\Delta p)^{2} h \cdot \nu d \Sigma & =2 \int_{Q} \Delta p\left(\sum_{i=1}^{n} \nabla h_{i} \cdot \nabla p_{x_{i}}\right) d Q+\int_{Q} \Delta p\left[\Delta h_{1}, \cdots, \Delta h_{n}\right] \cdot \nabla p d Q \\
& +\frac{1}{2} \int_{Q}\left[p \Delta p \Delta(\operatorname{div} h) d Q+\int_{Q} \Delta p \nabla p \cdot \nabla(\operatorname{div} h) d Q\right. \\
& -\int_{Q} F h \cdot \nabla p d Q-\frac{1}{2} \int_{Q} F p \operatorname{div} h d Q+b_{0, T}
\end{aligned}
$$




$$
b_{0, T}=\left[\left(p_{t}, h \cdot \nabla p\right)_{\Omega}\right]_{0}^{T}+\frac{1}{2}\left[\left(p_{t}, p \operatorname{div} h\right)_{\Omega}\right]_{0}^{T},
$$

where (2.22) can be rewritten (from (2.18)) more concisely as

$$
\begin{aligned}
\frac{1}{2} \int_{\Sigma}(\Delta p)^{2} h \cdot \nu d \Sigma & =\int_{Q} \Delta p \operatorname{div}\left[\left(H+H^{T}\right) \nabla p\right] d Q+\frac{1}{2} \int_{Q} p \Delta p \Delta(\operatorname{div} h) d Q \\
& -\int_{Q} F h \cdot \nabla p d Q-\frac{1}{2} \int_{Q} F p \operatorname{div} h d Q+b_{0, T}
\end{aligned}
$$

The analysis below will show a fortiori that the terms in identity (2.24) are well defined by establishing appropriate estimates thereof.

2.3. Analysis of terms involving $F$ and the boundary data $b_{0, T}$. The crucial term in (2.24) is the one involving $F h \cdot \nabla p$.

Proposition 2.4. Let the assumptions of Proposition 2.1 hold true.

(a) Then, the following identity is satisfied:

$$
\begin{aligned}
& -\int_{Q} F h \cdot \nabla p d Q-\frac{1}{2} \int_{Q} F p \operatorname{div} h d Q+b_{0, T}=-\left[(w, h \cdot \nabla p)_{\Omega}\right]_{0}^{T}-\frac{1}{2}\left[(w, p \operatorname{div} h)_{\Omega}\right]_{0}^{T} \\
& -\int_{0}^{T}\left(\tilde{G}_{2} \tilde{G}_{2}^{*} w_{t}, h \cdot \nabla p_{t}\right)_{\Omega} d t-\frac{1}{2} \int_{0}^{T}\left(\tilde{G}_{2} \tilde{G}_{2}^{*} w_{t}, p_{t} \operatorname{div} h\right)_{\Omega} d t
\end{aligned}
$$

where

$$
\begin{aligned}
& \left(\tilde{G}_{2} \tilde{G}_{2}^{*} w_{t}, h \cdot \nabla p_{t}\right)_{\Omega}+\frac{1}{2}\left(\tilde{G}_{2} \tilde{G}_{2}^{*} w_{t}, p_{t} \operatorname{div} h\right)_{\Omega} \\
& \quad=-\frac{1}{2}\left(\tilde{G}_{2} \tilde{G}_{2}^{*} w_{t}, p_{t} \operatorname{div} h\right)_{\Omega}-\left(p_{t}, h \cdot \nabla\left(\tilde{G}_{2} \tilde{G}_{2}^{*} w_{t}\right)\right)_{\Omega} \\
& \quad=\mathcal{O}\left(\left\|\tilde{G}_{2}^{*} w_{t}\right\|_{L_{2}\left(\Gamma_{1}\right)}\left\|p_{t}\right\|_{L_{2}(\Omega)}\right)
\end{aligned}
$$

with constant in $\mathcal{O}$ depending on $h$ and $\left\|A^{1 / 4} \tilde{G}_{2}\right\|$.

(b) The following estimate holds true for the right hand side of (2.25), with $E(t)$ as in (1.20) where $\epsilon>0$ is arbitrary:

$$
\begin{aligned}
& -\int_{Q} F h \cdot \nabla p d Q-\frac{1}{2} \int_{Q} F p \operatorname{div} h d Q+b_{0, T} \\
\geq & -C_{h}[E(T)+E(0)]-\frac{1}{\epsilon} C_{h} \int_{0}^{T}\left\|\tilde{G}_{2}^{*} w_{t}\right\|_{L_{2}\left(\Gamma_{1}\right)}^{2} d t-\epsilon \int_{0}^{T}\left\|p_{t}\right\|_{L_{2}(\Omega)}^{2} d t .
\end{aligned}
$$

Proof: (a) Recalling $F=-\tilde{G}_{2} \tilde{G}_{2}^{*} w_{t t}$ from (2.12) and integrating by parts in $t$, we obtain

$$
\begin{aligned}
& -\int_{Q} F h \cdot \nabla p d Q=\int_{0}^{T}\left(\tilde{G}_{2} \tilde{G}_{2}^{*} w_{t t}, h \cdot \nabla p\right)_{\Omega} d t \\
& \quad=\left[\left(\tilde{G}_{2} \tilde{G}_{2}^{*} w_{t}, h \cdot \nabla p\right)_{\Omega}\right]_{0}^{T}-\int_{0}^{T}\left(\tilde{G}_{2} \tilde{G}_{2}^{*} w_{t}, h \cdot \nabla p_{t}\right)_{\Omega} d t
\end{aligned}
$$


and using now $\tilde{G}_{2} \tilde{G}_{2}^{*} w_{t}=-w-p_{t}$ from $(2.9)$, we obtain from (2.28),

$-\int_{Q} F h \cdot \nabla p d Q+\left[\left(p_{t}, h \cdot \nabla p\right)_{\Omega}\right]_{0}^{T}=-\left[(w, h \cdot \nabla p)_{\Omega}\right]_{0}^{T}-\int_{0}^{T}\left(\tilde{G}_{2} \tilde{G}_{2}^{*} w_{t}, h \cdot \nabla p_{t}\right)_{\Omega} d t$

Similarly,

$-\int_{Q} F p \operatorname{div} h d Q+\left[\left(p_{t}, p \operatorname{div} h\right)_{\Omega}\right]_{0}^{T}=-\left[(w, p \operatorname{div} h)_{\Omega}\right]_{0}^{T}-\int_{0}^{T}\left(\tilde{G}_{2} \tilde{G}_{2}^{*} w_{t}, p_{t} \operatorname{div} h\right)_{\Omega} d t$.

Then (2.29) and (2.30) lead to (2.25). Finally, using the identity

$$
\int_{\Omega} v h \cdot \nabla \psi d \Omega=\int_{\Gamma} v \psi h \cdot \nu d \Gamma-\int_{\Omega} \psi h \cdot \nabla v d \Omega-\int_{\Omega} v \psi \operatorname{div} h d \Omega,
$$

a consequence of the divergence theorem, with $v=\tilde{G}_{2} \tilde{G}_{2}^{*} w_{t}$ and $\psi=p_{t}$ along with the B.C. $\left.p_{t}\right|_{\Gamma}=0$ from (2.11c), we readily verify identity $(2.26 \mathrm{a})$, from which estimate $(2.26 \mathrm{~b})$ follows at once by $(1.9)$.

(b) Estimate (2.27) follows readily from $(2.25),(2.26 \mathrm{~b})$, recalling $(2.13)$ and the definition (1.20) of $E(t)$.

2.4. Completion of the proof of Theorem 1.2: The radial field case and absence of geometrical conditions if $\Gamma_{0}=\phi$.

Step 1: We specialize to the radial field $h(x)=x-x_{0}$ as in the assumption

$$
H(x)=\text { Identity } ; \operatorname{div} h \equiv \operatorname{dim} \Omega=n ; \nabla(\operatorname{div} h) \equiv 0,
$$

so that the basic identity (2.22), or its more concise form (2.24), becomes

$$
\begin{aligned}
\frac{1}{2} \int_{\Sigma}(\Delta p)^{2} h \cdot \nu d \Sigma & =2 \int_{Q}(\Delta p)^{2} d Q-\int_{Q} F h \cdot \nabla p d Q \\
& -\frac{1}{2} \int_{Q} F p \operatorname{div} h d Q+b_{0, T}
\end{aligned}
$$

Next, Identity (2.21) and Remark 2.2 give

$$
\int_{Q}(\Delta p)^{2} d Q=\int_{Q} p_{t}^{2} d Q+\int_{Q} F p d Q-\left[\left(p_{t}, p\right)_{\Omega}\right]_{0}^{T}
$$

which inserted in (2.33) produces the identity

$$
\begin{aligned}
\frac{1}{2} \int_{\Sigma}(\Delta p)^{2} h \cdot \nu d \Sigma & =2 \int_{Q} p_{t}^{2} d Q-\int_{Q} F h \cdot \nabla p d Q-\frac{1}{2} \int_{Q} F p \operatorname{div} h d Q \\
& +2 \int_{Q} F p d Q-2\left[\left(p_{t}, p\right)_{\Omega}\right]_{0}^{T}+b_{0, T} .
\end{aligned}
$$

Summing up (2.33) and (2.35) results in

$$
\begin{aligned}
& \frac{1}{2} \int_{\Sigma}(\Delta p)^{2} h \cdot \nu d \Sigma=2 \int_{Q}\left[(\Delta p)^{2}+p_{t}^{2}\right] d Q \\
- & 2\left\{\int_{Q} F h \cdot \nabla p d Q+\frac{1}{2} \int_{Q} F p \operatorname{div} h d Q-b_{0, T}\right\}+2 \int_{Q} F p d Q-2\left[\left(p_{t}, p\right)_{\Omega}\right]_{0}^{T}
\end{aligned}
$$


Step 2: We now recall (2.15) for the first term on the right of (2.36); estimate (2.27) for the last term in $\{\cdot\}$ of $(2.36)$; and a similar estimate for the last two terms in (2.36) (which are, in fact, contained in (2.27)). We obtain for the right hand side (R.H.S.) of (2.36):

R.H.S. of $(2.36) \geq(2-\epsilon) \int_{0}^{T} E(t) d t-C_{h}[E(T)+E(0)]-\frac{C_{h}}{\epsilon} \int_{0}^{T}\left\|\tilde{G}_{2}^{*} w_{t}\right\|_{L_{2}\left(\Gamma_{1}\right)}^{2} d t$.

Recalling (1.18), we rewrite (2.37) as

$$
\text { R.H.S. of }(2.36) \geq(2-\epsilon) \int_{0}^{T} E(t) d t-2 C_{h} E(T)-C_{h, \epsilon} \int_{0}^{T} \int_{\Gamma_{1}}\left(\frac{\partial w}{\partial \nu}\right)^{2} d \Gamma d t .
$$

Step 3. From (1.7) or (1.12e) and (2.8), we have

$$
\left.\frac{\partial w}{\partial \nu}\right|_{\Sigma_{1}}=\left[\Delta A^{-1} w_{t}\right]_{\Sigma_{1}}=[\Delta p]_{\Sigma_{1}}
$$

so that with the definition of $\Gamma_{0}=\Gamma_{-}\left(x_{0}\right)$ given by $(1.22)$, we have for the left hand side (L.H.S.) of (2.36)

$$
\begin{aligned}
& \text { L.H.S. of }(2.36)=\int_{\Sigma}(\Delta p)^{2}\left(x-x_{0}\right) \cdot \nu d \Sigma \\
& \quad \leq \int_{\Sigma_{1}}(\Delta p)^{2}\left(x-x_{0}\right) \cdot \nu d \Sigma_{1} \leq C_{h} \int_{0}^{T} \int_{\Gamma_{1}}\left(\frac{\partial w}{\partial \nu}\right)^{2} d \Sigma_{1} .
\end{aligned}
$$

Combining (2.38) with (2.40), we obtain

$$
\begin{aligned}
C_{h, \epsilon} \int_{0}^{T} \int_{\Gamma_{1}}\left(\frac{\partial w}{\partial \nu}\right)^{2} d \Gamma d t & \geq(2-\epsilon) \int_{0}^{T} E(t) d t-2 C_{h} E(T) \\
& \geq\left[(2-\epsilon) T-2 C_{h}\right] E(T),
\end{aligned}
$$

where in the last step of (2.41) we have used the dissipative property (1.17) of $E(t)$. Taking $T$ sufficiently large in (2.41) yields the desired estimate (2.2). Theorem 1.2 is proved.

2.5. Final remarks on possible generalizations. In this section we elaborate on the possible generalizations pointed out in Remarks 1.2 and 1.3.

Concerning inequality (1.26). Let $h(x) \in\left[C^{3}(\bar{\Omega})\right]^{n}$ be a vector field, and let $H(x)$ be the $n \times n$ matrix with $(i, j)$-entry $\frac{\partial h_{i}}{\partial x_{j}}$ as in section 2.2 . If $h(x)$ is radial, then $H(x) \equiv$ identity. We then consider the following perturbation $H(x)$ of the identity matrix:

(i) Let the off-main diagonal terms $\frac{\partial h_{i}}{\partial x_{j}}, i \neq j$, be sufficiently small in the supnorm;

(ii) let the main diagonal terms satisfy the conditions that (sup over all $i$ and all $x \in \bar{\Omega})$

$$
\sup \left[\frac{\partial h_{i}}{\partial x_{i}}(x)-m\right] \equiv d
$$

be sufficiently small, for a constant $m>0$. 
Then, inequality (1.26) holds true, if $q \in H_{0}^{2}(\Omega)$ as assumed.

In fact, we may write

$$
\begin{gathered}
\Delta q\left(\sum_{i=1}^{n} \nabla h_{i} \cdot \nabla q_{x_{i}}\right)=m(\Delta q)^{2}+(\Delta q) Q(x) \\
Q(x)=\sum c_{i j}(x) q_{x_{i} x_{j}} \\
\int_{\Omega} \Delta q\left(\sum_{i=1}^{n} \nabla h_{i} \cdot \nabla q_{x_{i}}\right) d \Omega \geq m \int_{\Omega}(\Delta q)^{2} d \Omega-\int_{\Omega}|(\Delta q) Q| d \Omega .
\end{gathered}
$$

But for each $i, j$,

$$
\left\|q_{x_{i} x_{j}}\right\|_{L_{2}(\Omega)} \leq\|q\|_{H^{2}(\Omega)} \leq C\|q\|_{\mathcal{D}\left(A^{1 / 2}\right)}=c\left\|A^{1 / 2} q\right\|_{L_{2}(\Omega)}=c\|\Delta q\|_{L_{2}(\Omega)}
$$

since $q \in H_{0}^{2}(\Omega)=\mathcal{D}\left(A^{1 / 2}\right)$, with equivalent norms (see (1.3) and (1.5)), where $c$ is a constant of equivalence. Hence, from (2.44), (2.46), we obtain

$$
\int_{\Omega}|(\Delta q) Q| d \Omega \leq k \int_{\Omega}(\Delta q)^{2} d \Omega
$$

so that $(2.45),(2.47)$ imply

$$
\int_{\Omega} \Delta q\left(\sum_{i=1}^{n} \nabla h_{i} \cdot \nabla q_{x_{i}}\right) d \Omega \geq(m-k) \int_{\Omega}(\Delta q)^{2} d \Omega .
$$

The constant $k$ depends on $\sup \left|\frac{\partial h_{i}}{\partial x_{j}}\right| ; i \neq j$; on $d$ in (2.42); on $c$ in (2.46); and if these quantities are sufficiently small with respect to $m$, we may obtain $m-k=\rho>0$ as desired. This situation occurs, in particular, for linear fields as in (1.24), (1.25) of Remark 1.2.

Modifications of the proof of Section 2 for a vector field satisfying inequality (1.26). We return to the basic identity (2.22). If inequality (1.26) holds true, we readily find for the right hand side (R.H.S.) of (2.22):

$$
\begin{aligned}
\text { R.H.S. of }(2.22) & \geq(2 \rho-\epsilon) \int_{Q}(\Delta p)^{2} d Q-\frac{M_{h}}{\epsilon} \int_{Q}|\nabla p|^{2} d Q \\
& -\int_{Q} F h \cdot \nabla p d Q-\frac{1}{2} \int_{Q} p \operatorname{div} h d Q+b_{0, T}
\end{aligned}
$$

where the constant $M_{h}$ is defined in (1.29) (and is zero if $h$ is linear as in (1.24), $(1.25))$. The proof now proceeds as in section 2 and yields the inequality

$$
\begin{aligned}
C_{h, \epsilon} \int_{0}^{T} \int_{\Gamma_{1}}(\Delta p)^{2} d \Sigma+\frac{M_{h} T}{\epsilon}\||\nabla p|\|_{C\left([0, T] ; L_{2}(\Omega)\right)}^{2} \\
\geq\left[(2 \rho-\epsilon) T-2 C_{h}\right]\left\|\left\{p_{0}, p_{1}\right\}\right\|_{\mathcal{D}\left(A^{1 / 2}\right) \times L_{2}(\Omega)}^{2}
\end{aligned}
$$

counterpart of (2.41) (recall that $\frac{\partial w}{\partial \nu}=\Delta p$ on $\Sigma_{1}$, by (2.39)), where we have also used (1.18) for $t=T$ and (2.13), (2.14); where we now have an additional (lower order) term $|\nabla p|$. We absorb this as follows. 
Lemma 2.1. Inequality (2.50) implies that there is a constant $C_{T}$ such that

$$
\||\nabla p|\|_{C\left([0, T] ; L_{2}(\Omega)\right)}^{2} \leq C_{T} \int_{0}^{T} \int_{\Gamma_{1}}(\Delta p)^{2} d \Sigma .
$$

Proof: The proof follows similar arguments e.g., [6-7], [9-10], etc., with the following novelty: The contradiction argument

$$
\begin{gathered}
\int_{0}^{T} \int_{\Gamma_{1}}\left(\Delta p_{n}\right)^{2} d \Sigma \rightarrow 0 \\
\left\|\left|\nabla p_{n}\right|\right\|_{C\left([0, T] ; L_{2}(\Omega)\right)} \equiv 1,
\end{gathered}
$$

for solutions $p_{n}$ of the non-homogeneous $p$-problem $(2.11)$, leads in the usual way via $(2.50)$ to the result that $\left\{p_{n}\right\}$ is uniformly bounded in $L_{\infty}\left(0, T ; \mathcal{D}\left(A^{1 / 2}\right)\right)$, and hence, by compactness [17],

$$
p_{n} \rightarrow \text { limit function } \tilde{p}, \text { strongly in } L_{\infty}\left(0, T ; \mathcal{D}\left(A^{1 / 4}\right)\right),
$$

so that from $(2.52),(1.4)$

$$
\||\nabla \tilde{p}|\|_{C\left([0, T] ; L_{2}(\Omega)\right)}=1 .
$$

Now, the limit $\tilde{p}$ satisfies $\left.\Delta \tilde{p}\right|_{\Sigma_{1}}=0$ by $(2.51)$, and hence the corresponding right hand side function $\tilde{F}$ in $(2.11),(2.12)$ becomes $\tilde{F}=\tilde{G}_{2}\left[\Delta \tilde{p}_{t}\right]_{\Sigma_{1}} \equiv 0$. Thus, the limit problem for $\tilde{p}$ becomes homogeneous on the right hand side (as in the corresponding exact controllability question):

$$
\begin{array}{ll}
\tilde{p}_{t t}+\Delta^{2} \tilde{p}=0 & \text { in } Q ; \\
\left.\tilde{p}\right|_{\Sigma}=\left.\frac{\partial \tilde{p}}{\partial \nu}\right|_{\Sigma}=0 & \text { in } \Sigma \\
\left.\Delta \tilde{p}\right|_{\Sigma_{1}}=0 & \text { in } \Sigma_{1} .
\end{array}
$$

It is here that the uniqueness property (1.28) is invoked to obtain $\tilde{p} \equiv 0$ in $Q$, a contradiction with (2.54). Thus, (2.51) is proved.

The rest of the proof proceeds as in section 2, following (2.41).

Remark 2.3. Finally, we remark that if, instead of criterion (2.1), one uses the equivalent criterion (Datko's Theorem):

$$
\int_{0}^{\infty} E(t) d t \leq C E(0)
$$

then absorption of the lower order terms can be done as in [1, Theorem $1.3 \mathrm{~b}]$; but this requires that the imaginary axis belongs to the resolvent set $\rho(\mathcal{A})$ of $\mathcal{A}$. Hence, in this case, the elliptic uniqueness property (1.21) is needed.

\section{REFERENCES}

[1] J. Bartolomeo and R. Triggiani, Uniform energy decay rates for the Euler-Bernoulli equations with feedback operators in the Dirichlet/Neumann boundary conditions, SIAM J. Math. Analysis, to appear. 